NSSIR $86-3389$

\title{
A Near-Optimal Starting Solution for Polynomial Approximation of a Continuous function in the $\mathrm{L}_{1}$ Norm
}

P. D. Domich

Senter for Applied Marhematics

National Engineering Laboratory

National Bupeau of Stanjards

Gaithersburg, M0 20895

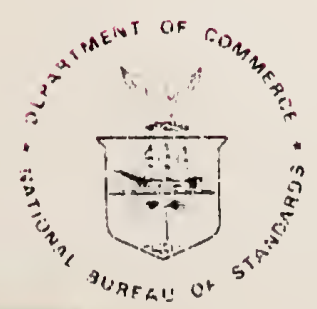



NBSIR 86-3389

\section{A Near-Optimal Starting Solution for Polynomial Approximation of a Continuous Function in the $L_{1}$ Norm}

P. D. Domich

Center for Applied Mathematics National Engineering Laboratory National Bureau of Standards

Gaithersburg, MD 20899

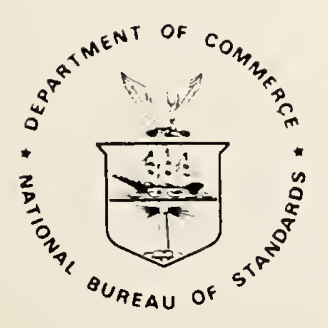

U.S. DEPARTMENT OF COMMERCE, Malcolm Balarige. Secretary NATIONAL BUREAU OF STANDARDS. Ernest Ambler. Director 


\section{ABSTRACT}

This paper presents a nethod of selecting a near-optimal starting solution for a large class of discrete polynomial approximation problems in the $\mathrm{L}_{1}$ norm. While it is possible to prove the optimality of these advanced starting solutions for only a small class of continuous polynomial approximation problems, empirical evidence indicates the starting bases will be nearly optimal for a much larger class of discrete problems. This paper presents the method used to determine the starting basis and a heuristic justification backed by empirical results supporting its use. 



\section{INTRODUCTION}

The investigation of a near-optimal starting solution for discrete polynomial approximation problems in the $L_{1}$ norm resulted from a study to develop a methodology for comparing and evaluating mathematical programming software. ${ }^{1}$ In this study four $L_{1}$ approximation algorithms $2,3,4,5$ were tested on a large set of problems with diverse problem structures and characteristics. Included were 80 problems used to test code performance in the polynomial approximation of a continuous function, a specific type of approximation problem. In these problems, the continuous function being approximated, the degree of the approximating polynomial, and the number of observation points were independently varied. In all problems, the observations were equally spaced over the sane interval of approximation, $[0,1]$.

When the interpolation points defining the best $\mathrm{L}_{1}$ solution were tabulated, with the degree of the polynomial and the number of observations fixed, but varying the functional form, a recurrent pattern emerged which indicated that the points of interpolation remained relatively stationary within the normalized interval regardless of the functional form being approximated. This pattern suggested a method of selecting a starting basis which would be nearly optimal for a large number of approximation problems. 
After further study, an analytic expression was identified that can be used to calculate a feasible starting solution which, when used in the continuous analog of the discrete approximation problem, is the optimal solution in the $\mathrm{L}_{1}$ norm. 6 Calculating this starting solution and using it in the discrete approximation problem also produced impressive empirical results, both in terms of its relative distance to the optimal solution and also in the marked reduction in the number of iterations needed to reach the optimal solution. Furthermore, the integration of this method of selecting the starting basis into existing $\mathrm{L}_{1}$ algorlthms is easy. An explanation of the success of this method can be found in the underlying approximation theory and is presented in the next two sections. The final section reports the results of using this near-optimal starting basis on a large set of test problems.

\section{THE DISCRETE POLYNOMIAL APPROXIMATION PROBLEM}

The general form of the discrete $\mathrm{L}_{1}$ approximation problem can be formulated as follows.

Given $n$ sets of observations on a dependent variable $y_{1}$ and a single 1ndependent variable $x_{1}$

$$
\left\{x_{1}, y_{1}\right\}
$$

determine $\mathrm{m}+1$ parameters

$$
\bar{\beta}=\beta_{0}, \beta_{1}, \beta_{2}, \ldots, \beta_{m},
$$

which mintmize

$$
z=\sum_{i=1}^{n}\left|y_{i}-\sum_{j=0}^{m} \beta_{j} x_{i}^{j}\right| .
$$


This is the polynomial approximation of a continuous function when $y_{i}=f\left(x_{1}\right)$, where $f(x)$ is a continuous function defined over an approximation interval $I$. Introducing variables $d$ and $d$, corresponding to the positive and negative deviation, the approximation problem can be posed as the following linear programming problem.

$$
\text { Minimize } \sum_{i=1}^{n} d_{i}^{+}+d_{i}^{-} \text {, }
$$

subject to

$$
\begin{aligned}
& d_{i}^{+}-d_{i}^{-}+y_{i}-\sum_{j=0}^{m} \beta_{j} x_{1}^{j}=0, \\
& d_{i}^{+} \geq 0, d_{i}^{-} \geq 0 \text { for } i=1,2,3, \ldots, n \text {. }
\end{aligned}
$$

For this class of problems it is not unreasonable to assume the problems are of full rank (Independent columns) and overdetermined (the number of observations strictly greater than $m$, the number of $\beta$ parameters in the problem). A further condition necessary for one to "predict" the best solution to any problem of this type is that the solution be unique. When the uniqueness condition is satisfied and the number of observations sufficlently large, the starting solution we suggest is very close to the optimum. Unfortunately, unfqueness cannot be guaranteed for certain approximation problems. 
III. FUNCTIONAL ANALYSIS AND PROPERTIES OF $L_{1}$ APPROXIMATION OF A CONTINUOUS FUNCTION

This section presents the theoretical bas1s behind a unique best $\mathrm{L}_{1}$ approximation solution for a special class of continuous functions. The section begins by presenting several properties of the polynomlal approximation of a continuous function in the $\mathrm{L}_{1}$ norm. These properties establish the existence, uniqueness and optimality of a solution. A description of a class of functions which have these properties is developed and 1 w w11 be shown that the starting basis we propose w1ll be the optimal $\mathrm{L}_{1}$ solution. An alternate representation of the optimal $L_{1}$ solution, which serves as a useful gulde in determining the "goodness" of an approximation is also presented as is a method for calculating the starting basis easily and efficiently.

Since the best $L_{1}$ polynomial approximation of degree m to a continuous function, $f(x)$, intersects the function $1 n \cdot$ at least $m+1$ points ${ }^{7}$, we will examine the additional characteristics a function must possess to insure the uniqueness of the optimal solution. The first assumption on the function requires the existence of a non-trivial approximation problem, i.e., $f(x)$ is different from the $\mathrm{L}_{1}$ approximation almost everywhere. Had this not been assumed, any $m+1$ observations would have defined the best $L_{1}$ approximation. By requiring that $f(x)$ have a $m+1^{\text {st }}$ derivative that is continuous and non-constant, a nontrivial approximation problem is guaranteed. We characterize any such function as a "higher-order function" than the polynomial of degree m. Th1s is closely analogous to the term "higher-order polynomials" though more general. 
The uniqueness of the final solution and ultimately the optimality of the suggested starting basis is established by making one further requirement on the derivatives of $f(x)$. If the $n+1^{\text {st }}$ derivative, $f^{m+1}(x)$, is nonvanishing over the approximation interval, then it will be shown that the best $\mathrm{L}_{1}$ polynomial approximation to $f(x)$ will intersect at exactly $m+1$ observations and, therefore, will be unique. With this condition satisfied, one can then find the $m+1$ observations which define the optimal $\mathrm{L}_{1}$ approximation to the continuous problem, evaluate the function at these points and enter these observations to the discrete problem basis. Solving the corresponding $(m+1) \cdot(m+1)$ system of equations defined by this new basis will locate a near-optimal $L_{1}$ solution to the discrete problem.

The following examples and subsequent theorems will solidify the theoretical underpinnings bounding the number of intersections between the continuous function and the best approximating polynomial. First consider the problem of locating all the possible intersections between a second-order polynomial $\mathrm{P}_{2}(\mathrm{x})$ and a first-order polynomial $\mathrm{p}_{1}(\mathrm{x})$, (shown graphically in figure 1 ).

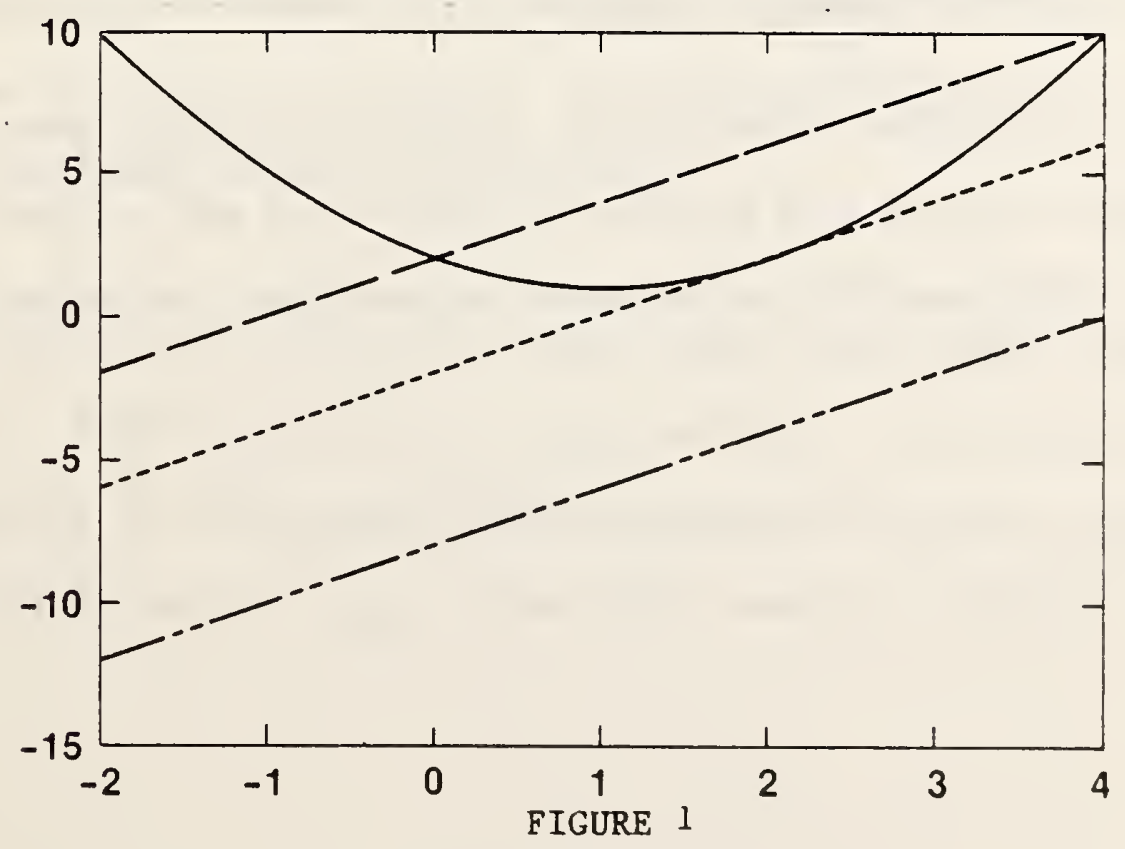


Three possible situations exist: first, $p_{1}(x)$ and $p_{2}(x)$ fail to intersect in $[a, b]$; second, $p_{1}(x)$ and $p_{2}(x)$ are tangent which will produce only one point of intersection; or finally, $p_{1}(x)$ and $p_{2}(x)$ intersect at two points. The problem of locating the points of intersection of $p_{1}(x)$ and $p_{2}(x)$ is equivalent to that of finding all possible roots of a residual function defined as their difference in $[a, b]$,

$$
r(x)=p_{2}(x)-p_{1}(x)=0, x \in[a, b]
$$

Since $r(x)$ is binomial, it can have at most two real-valued roots. This argument can easily be extended for any two polynomlals, $p_{m}(x)$ and $p_{m+1}(x)$, of degree $\mathrm{m}$ and $\mathrm{m}+1$ respectively, with no loss of generality.

The task of establishing the number of intersections of $p_{m}(x)$ and a polynomial of degree $m+k, P_{m+k}(x)$, for $k>1$, or any function of higher order is slightly more difficult. Again, the behavior of the derivatives will determine the number of roots the residual function

$$
r(x)=p_{m+k}(x)-p_{m}(x)
$$

will have over $[a, b]$. By assuming a restriction on $p_{m+k}$, it is again possible to limit the number of roots $r(x)$ may have to at most $m+l$ and therefore define a larger class of problems which will have a unique best $\mathrm{L}_{1}$ solution. 
Lemma 1. Suppose a function $f(x)$ is continuously differentiable at least $k$ times in an interval $[a, b]$. Suppose further that $f^{k}(x)>0(\langle 0)$ in $[a, b]$. Then $f^{k-1}(x)$ is strictly monotone increasing (decreasing) in $[a, b]$, and there is only one possible zero of $\mathrm{f}^{\mathrm{k}-1}(\mathrm{x})$ in $[\mathrm{a}, \mathrm{b}]$.

Together with the following theorem it will be possible to establish upper bounds on the number of real-valued roots for each successively lower-ordered derivative.

Theorem 1 [McCormick ${ }^{8}$ ]. Suppose a function $f(x)$ is continuously differentiable at least $k$ times in an interval $[a, b]$. Suppose that $f^{k}(x)$ has $q$ zeros in that interval and that they are known and ordered as $a \leq z_{1}<z_{2}<z_{3} \ldots<z_{q} \leq b$. Then $f^{k-1}(x)$ is strictly monotone in $\left[a, z_{1}\right],\left[z_{1}, z_{1+1}\right]$, (for $\left.1=1, \ldots, q-i\right)$, and $\left[z_{q}, b\right]$. There are at most $q+1$ zeros of $f^{k-1}(x)$ in $[a, b]$. Specifically, there may be one in $\left[a, z_{1}\right]$, one in each of $\left[z_{1}, z_{1+1}\right]$, (for $1=1, \ldots, q-1$ ) and one in $\left[z_{q}, b\right]$. If $f^{k-1}(a) \cdot f^{k-1}\left(z_{1}\right)>0$ there is no zero in that interval, otherwise there is exactly one there. If (for $1=1,2, \ldots, q-1) f^{k-1}\left(z_{i}\right) \cdot f^{k-1}\left(z_{i+1}\right)>0$, there is no zero in that interval. If $\mathrm{f}^{\mathrm{k}-1}\left(\mathrm{z}_{\mathrm{q}}\right) \cdot \mathrm{f}^{\mathrm{k}-1}(\mathrm{~b})>0$, there is no zero in $\left[z_{q}, b\right]$. Otherwise there is exactly one.

Proof. Since $f^{k}\left(z_{1}\right)=f^{k}\left(z_{1+1}\right)=0$ and there are no zeros between $z_{1}$ and $z_{1+1}$ then $f^{k}(x)>0$ for all $x$ in $\left(z_{1}, z_{1+1}\right)$, or $f^{k}(x)<0$ in that interval. Thus the hypotheses of the previous lemma are satisfied and the appropriate conclusion follows for $\left[z_{1}, z_{1+1}\right]$. The other cases are identical. 
Thus, for any function $f(x)$ with a nonvanishing and monotonic $(m+1)^{\text {st }}$ derivative Theorem 1 can be applied successively to construct upper bounds to each successively lower-order derivative until a upper bound of m+l can be established for the number of real valued roots to $f(x)$ over the interval $[a, b]$. The upper bound on the number of roots of the residual function $r(x)$ is, therefore, dependent only on the behavior of $f^{m+l}(x)$ since the $(m+1)^{\text {st }}$ derivative of the approximating polynomial $\mathrm{P}_{\mathrm{m}}(\mathrm{x})$ is zero valued over the real I1ne, thus

$$
\begin{aligned}
r^{m+1}(x) & =f^{m+1}(x)-p_{m}^{m+1}(x) \\
& =f^{m+1}(x)
\end{aligned}
$$

Under the assumptions above and by requiring that the associated approximation problem is of full rank, the existence of a unique optimal solution to the discrete polynomial approximation problem defined by exactly $m+1$ observations is guaranteed. The final question remains. How to select the $m+1$ observations close to the optimal solution?

As shown in Rivilin ${ }^{10}$ and Rice ${ }^{11}$, there exists a large class of continuous functions for which the optimal $L_{1}$ solution in the continuous approximation problem can be determined a prior whenever it is known a priori that the optimal solution is unique and that the interpolation of these two functions occur at $m+1$ points. The necessary and sufficient requirements on $f(x)$ for this to occur are 1) $f(x)$ is continuous, 2) the difference of the function $f(x)$ and the $L_{l}$ approximating polynomial $P_{m}(x)$ is different from zero almost everywhere on $[a, b], 3)$ the residual function, $r(x)=f(x)-P_{m}(x)$, changes in sign at a unique set of $m+1$ points in the interval $[a, b]$. These points of interpolation are determined in the following theorem. 
Theorem 2: If $f(x)$ is continuous and differentiable, and $f(x)$ and $p_{m}(x)$ intersect $m+1$ times in the interval $[-1,1]$, then the least $L_{1}$ approximation is the unique polynomial $\mathrm{p}_{\mathrm{m}}^{*}(\mathrm{x})$ which satisfies

$$
\mathrm{P}_{\mathrm{m}} *\left(\cos \frac{j \Pi}{\mathrm{m}+2}\right)=f\left(\cos \frac{j \Pi}{m+2}\right) \text {, for } j=1,2, \ldots, m+1.11
$$

This optimal set of observations are the roots of the $\mathrm{m}^{\text {th }}$ degree Chebyshev polynomial of the second kind, $\operatorname{Um}(x)$. The explicit expression for this family of polynomials is listed below:

$$
\mathrm{U}_{\mathrm{m}}(\mathrm{x})=\sum_{i=1}^{\mathrm{m} / 2}(-1)^{i} \frac{(\mathrm{m}-\mathrm{i}) !}{i !(\mathrm{m}-2 i) !}(2 \mathrm{x})^{\mathrm{m}-2 i}
$$

or equivalently

$$
U_{m}(\cos \theta)=\frac{\sin (m+1) \theta}{\sin \theta} \text { for } x=\cos \theta \text {; }
$$

which have the following recurrence relation

$$
U_{\mathrm{m}+1}(\mathrm{x})=2 \mathrm{xU}_{\mathrm{m}}(\mathrm{x})-\mathrm{U}_{\mathrm{m}-1}(\mathrm{x}) \cdot 12
$$

The first four of these polynomials are: 


$$
\begin{aligned}
& \mathrm{U}_{0}(\mathrm{x})=1, \\
& \mathrm{U}_{1}(\mathrm{x})=2 \mathrm{x}, \\
& \mathrm{U}_{2}(\mathrm{x})=4 \mathrm{x}^{2}-1 ; \\
& \mathrm{U}_{3}(\mathrm{x})=8 \mathrm{x}^{3}-4 \mathrm{x}
\end{aligned}
$$

The series in which these polynomials are used in approximation to a continuous function $f(x)$,

$$
f(x)=\sum_{j=0}^{k} b_{j} U_{j}(x),
$$

are called Chebyshev Series and if

$$
\lim _{1 \rightarrow \infty} b_{1}=0
$$

the series is termed a Chebyshev series expansion of the function $f(x)$.

Chebyshev series expansions have long been known to converge very quickly to the target function compared to other series approximations and can be a useful tool in determining the significance of the neglected term in the $\mathrm{L}_{1}$ polynomial approximation. Generating the equivalent Chebyshev representation to the best $L_{1}$ approximating polynomial provides the user with information on the relative significance of the last term of the Chebyshev representation. When the coefficient of the higher order terms are very close to zero, the user can assume little accuracy w111 be gained by increasing the degree of the approximating polynomial. Conversely, if the last term of the Chebyshev approximation are of the same order as the previous coefficients, attempts to include more terms may provide a better approximation, or give the user an indication that the target function $f(x)$ may not have a converging Chebyshev serles expansion and an alternate approximation method should be used. 
IV. GENERATING THE STARTING BASIS

The method used in calculating the starting basis involves determining the zero-valued points of the Chebyshev polynomial. Using the trigonometric representation of the Chebyshev polynomials,

$$
U_{m}(x)=U_{m}(\cos \theta)=\frac{\sin (m+1) \theta}{\sin \theta}
$$

it is clear that the zero values of $U_{m}(x)$ occur at

$$
\mathrm{x}_{i}^{*}=\cos \underset{\mathrm{m}+1}{\mathrm{k} \pi} \text { for } \mathrm{k}=1,2, \ldots, \mathrm{m} \text { and }-1 \leqslant \mathrm{x}_{1} \leqslant 1 \text {. }
$$

In our work, the zero-valued points of $U_{m}(x)$ were translated into the interval of approximation, evaluated by the continuous function, and entered as constraints to the problem. Results of using these points as a starting basis are presented in the next section.

\section{COMPUTATIONAL RESULTS}

In our experiment, 256 approximation problems were used in testing the performance of the advanced starting method described. All problems were produced by an $\mathrm{L}_{1}$ polynomial approximation test-problem generator ${ }^{13}$ using eight different continuous functions, varying both in form and difficulty (see Table la of the append $(x)$. The problems generated ranged in degree from 3 to 10 , with $100,200,400$, and 800 observations over the closed interval $[0,1]$. Each 
problem was solved twice. One run of all 256 problems did not use the advanced solution method but included together with the sorted equidistant observations, the $m+1$ generated observations $\left\{x_{1}{ }^{*}\right\}$ used in the new starting solution method, thereby guaranteeing identical problems in both runs. The 256 problems were then rerun. The observations $\left\{x_{1}^{*}\right\}$ now formed the first $m+1$ constraints to the problem and were selected to enter the starting basis by the $\mathrm{L}_{1}$ code on the first m+1 pivots. The method used to solve all problems was double-precision $\mathrm{L}_{1}$-approximation algorithm developed by $\mathrm{N}$. N. Abdelmalek. ${ }^{14}$

In this section, the results from solving the problem with the new starting solution method are compared to results from the original method. Two performance measures were used in the comparison. One measured the distance between the objective function value $Z$ at the near-optimal starting point, and the optimum objective function value $Z^{*}$, normalized by the distance from the starting objective function value, $z^{s}$ (where $\bar{\beta}=0$ ), to $z^{*}$. or, mathematically, the normalized distance is

$$
D_{i}=\frac{z_{i}-z_{i}{ }^{*}}{z^{s}-z_{i}{ }^{*}} \quad 1=1,2, \ldots, 256 \text {. }
$$

A second measure was difference in iteration count between using the nearoptimal "starting basis" and the starting basis defined by $\bar{\beta}=0$. 
The results in measuring the performance of the advanced solution method by the normalized objective function distance are given in Table 2 of the appendix and summarized in Figure 2.*

Advanced Starting Solution Results

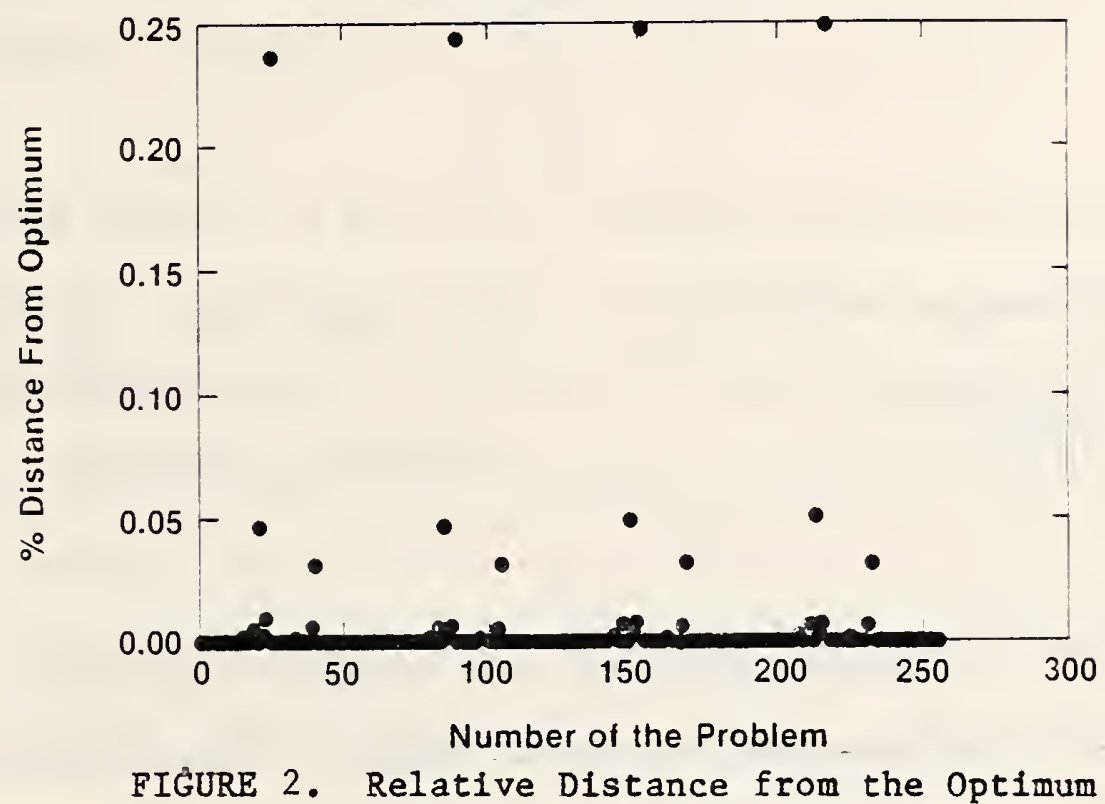

of the total 256 problems, only 12 were more than 1 percent from the optimum. Closer examination of the outliers reveals that all outliers of magnitude greater than 1 percent are related to problems produced by two of the eight continuous functions being approximated. Closer investigation reveals that the distance $D$ is worst in the approximations made by polynomials of lower degree. The functions (illustrated in Figures 3 and 4 ) connected with these outlier values are highly nonlinear and approximations by lesser degree polynomials are very poor.

\footnotetext{
${ }^{*}$ The regular pattern of the outliers in Figure 2 illustrate that problems of a specific function and degree are the same relative distance from the optimum for $100,200,400$, and 800 observations.
} 


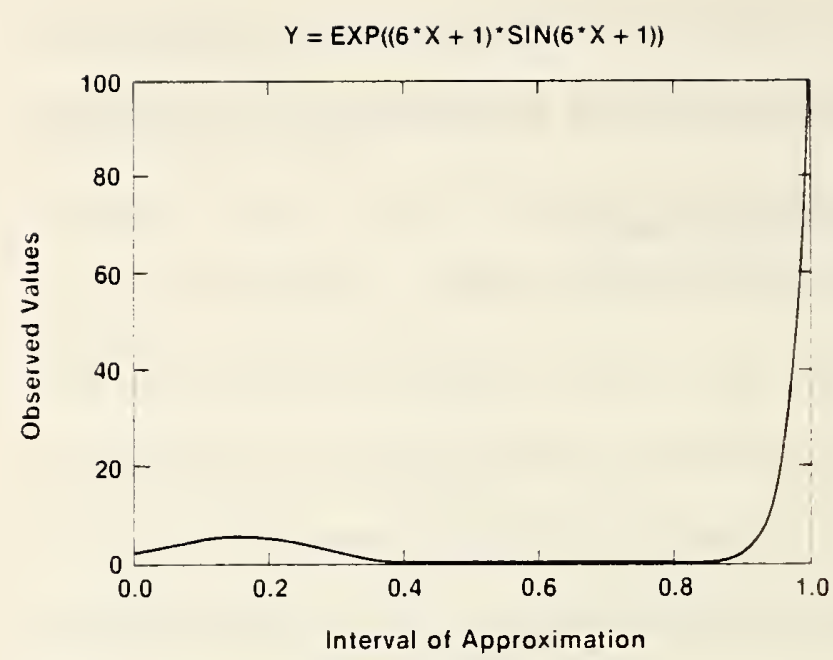

FIGURE 3. FUNCTION 3:

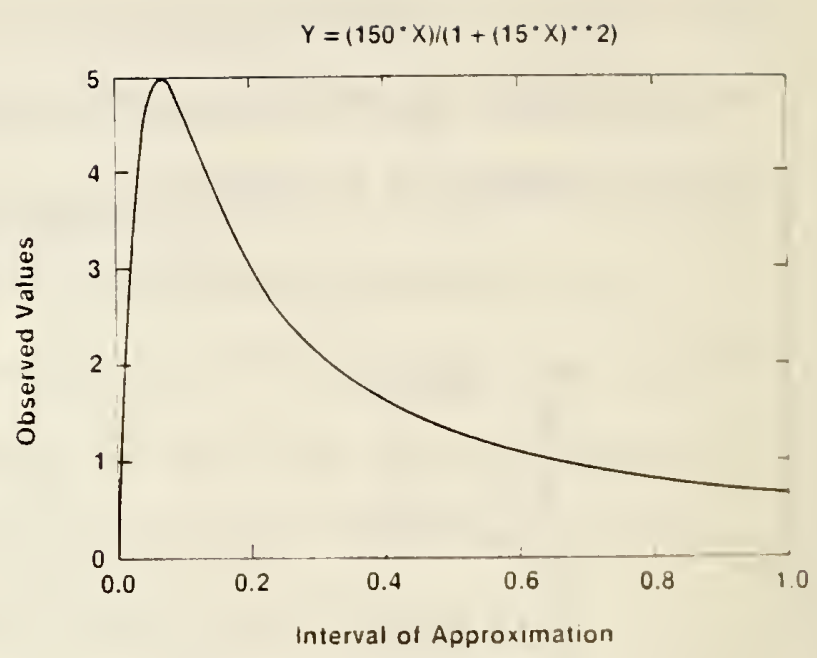

FIGURE 4. FUNCTION 5:

Though selection of a low degree polynomial was unwise by the user, the startIng basts did reduce the number of 1terations required to solve these problems In 13 of the 16 cases, as shown In Table 1. Similar data for problems of 200 , 400 , and 800 observations are given in Table $2 a$ of the appendix.

\begin{tabular}{lcccccccc}
\hline $\begin{array}{l}\text { DEGREE OF } \\
\text { POLYNOMIAL }\end{array}$ & 10 & 9 & 8 & 7 & 6 & 5 & 4 & 3 \\
$\begin{array}{l}\text { STARTING } \\
\text { METHOD }\end{array}$ & NO YES & NO YES & NO YES & NO YES & NO YES & NO YES & NO YES & NO YES \\
\hline
\end{tabular}

FUNCTIION \begin{tabular}{llllllllllllllllll}
\hline 3 & 28 & 14 & 42 & 27 & 19 & 14 & 24 & 8 & 20 & 23 & 24 & 19 & 13 & 11 & 14 & 15
\end{tabular}

FUNCTION \begin{tabular}{llllllllllllllllll}
\hline 5 & 26 & 15 & 19 & 14 & 24 & 8 & 18 & 7 & 14 & 6 & 19 & 17 & 13 & 10 & 23 & 31
\end{tabular}

TABLE 1. ITERATIONS NEEDED TO OBTAIN AN OPTIMAL SOLUTION (NUMBER OF OBSERVATIONS $=100)$. 
Increasing the degree of the approximating polynomial, in these outlier cases, did reduce the $D_{i}$ values substantially, with 4 of the 16 problems listed above having an advanced starting objective function value identical to the optimal, to 8 significant digits.

Over all 256 problems, a total of 62 problems using the advanced method were at the optimum, requiring no additional pivots for an accuracy of $10^{-8}$. A total of 5558 iterations were necessary to solve all 256 problems using the standard method. Solving the 256 problems using the starting method reduced the total number of iterations needed to solve all problems by 2028 , a reduction of 36 percent. In all, 241 problems had a reduced iteration count, 2 marked no change, and 13 increased.

In problems where the degree of the polynomial was overspecified, a degenerate problem much more difficult to solve, is produced. We noted several problems of this type which had an increased iteration count but also had a very small normalized distance measure $D_{i}$. This suggests there are many other solutions with objective function values very close to the optimal solution. This could force the $\mathrm{L}_{1}$ problem solver to test a large number of solutions of nearly identical objective function value before obtaining the optimal objective function value. Solving these problems with a polynomial of lesser degree improves iteration count, and in several cases required no additional pivots. From this we can infer that in the problems where a lesser degree polynomial fit the function quite well, the Chebyshev expansion converged very quickly, thereby producing a good approximation to the problem. Inferences drawn from the results such as those described above may provide the user insight into 
the problem concerning "goodness of fit" and "sufficlency of degree" of the approximating polynomial.

The results of this computational study indicate ways in which $\mathrm{L}_{1}$-approximation codes used for polynomial approximation problems can be improved. They are collected below.

1. Use the zeroes of the Chebyshev polynomials as the observations in the starting basis.

2. Convert the $\mathrm{L}_{1}$ polynomial coefficients into the coefficlents of the equivalent Chebyshev polynomial representation to provide the user with 1nformation on the rate of convergence of the Chebyshev terms and the correctness of the user's degree specification. 
[1] Domich, P. D., Hoffman, K. L., Jackson, R. H. F., Saunders, P. B., and Shier, D. R., Evaluation of $\mathrm{L}_{1}$ Codes Using Polynomial Approximation Problems, unpublished working paper, National Bureau of Standards.

[2] Abdelmalek, N. N., An Efficient Method for the Discrete Linear $\mathrm{L}_{1}$ Approximation Problem, Mathematics of Computation, 29, 844-850 (1975).

[3] Armstrong, R. D. and Frome, E. L., A Comparison of Two Algorithms for Absolute Deviation Curve Fitting, Journal of the American Statistical Association, 71 , No. 354, 328-330 (1976).

[4] Barrodale, I. and Roberts, F. D. K., An Improved Algorithm for Discrete $\mathrm{L}_{1}$ Linear Approximation, SIAM Journal on Numerical Analysis, 10 , No. 5, 839-848 (1973).

[5] Bartels, R. H., Conn, A. R., and Sinclair, J. W., Minimization Techniques for Plecewise Differentiable Functions: The $\mathrm{L}_{1}$ Solution to an Overwhelmed Linear System, SIAM Journal on Numerical Analysis, 15 , No. $2,224-241$ (1978).

[6] Rivlin, T. J., An Introduction to the Approximation of Functions (Blalsdel1 Publishing. Company, Waltham, MA, pg. 73, 1969).

[7] Barrodale, I. and Roberts, F. D. K., An Improved Algorithm for Discrete $\mathrm{L}_{1}$ Linear Approximation, SIAM Journal on Numerical Analysis, 10 , No. 5 , pg. 839 (1973).

[8] McCormick, G. P., Finding the Global Minimum of a Function of One Variable Using the Method of Constant Signed Higher Order Derivatives, GWU Technical Report T-411, The George Washington University, 1979.

[9] Rivlin, T. J., An Introduction to the Approximation of Functions, (Blaisdel1 Publishing Company, Waltham, MA, pg. 73, 1969).

[10] Rice, J. R., The Approximation Functions, 1 (Addison-Wesley, Reading, MA, page 106,1964$)$.

[11] Rivlin, T. J., An Introduction to the Approximation of Functions, (Bla1sde11 Publishing Company, Waltham, MA, pg. 73, 1969).

[12] Davis, P. I., Interpolation and Approximation (Blaisdell Publishing Company, Waltham, MA, pg. 366, 1969).

[13] Domfch, P. D., Lawrence,J., and Shier,D.R. Generators for Discrete Polynomial L 1 Approximation Problems, Journal of Research, National Bureau of Standards, Vol. 84, No. 6, November-December 1979.

[14] Abdelmalek, N. N., An Efficient Method for the Discrete Linear L Approximation Problem, Mathematics of Computation, 29, 844-850 (1975). 

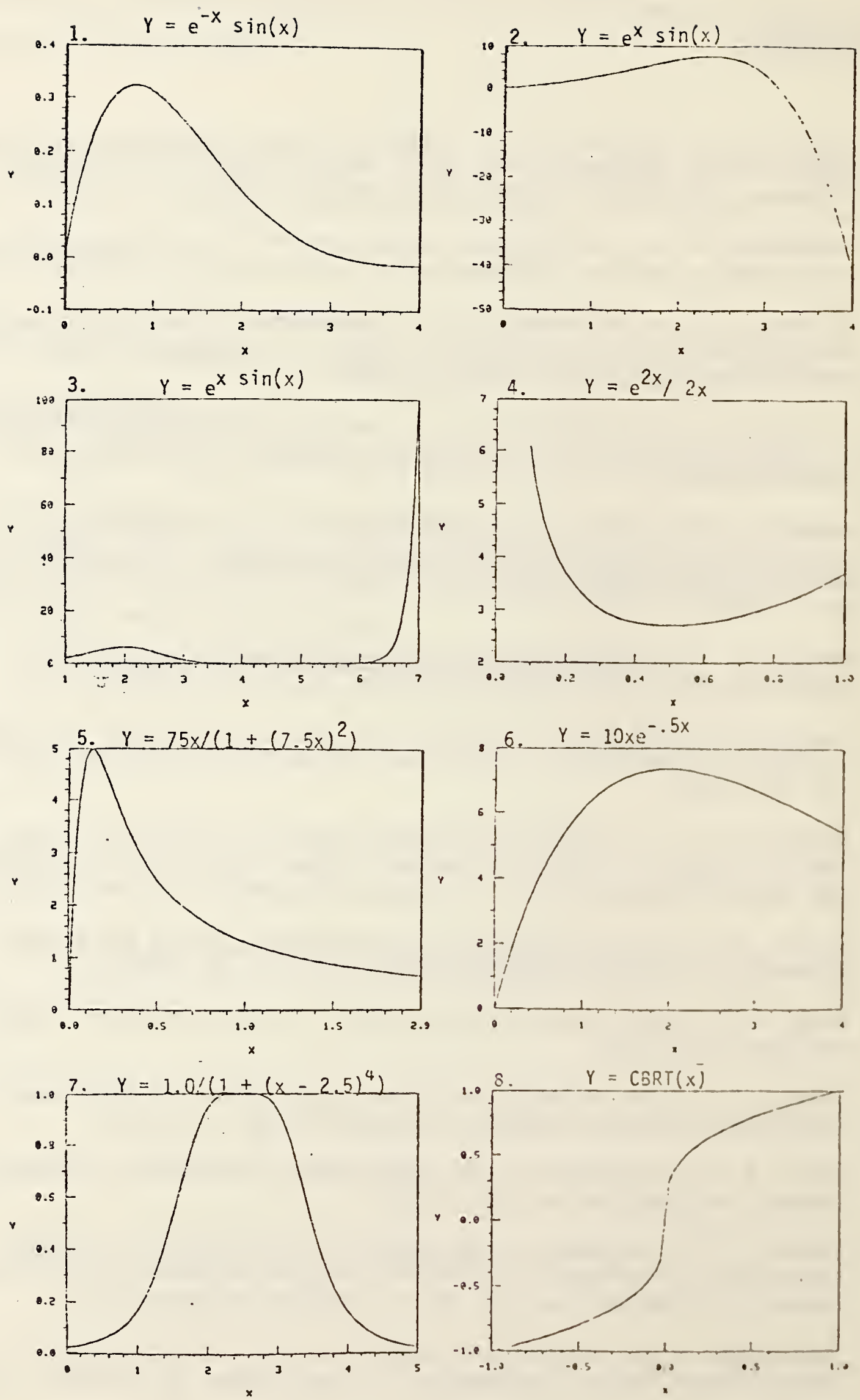


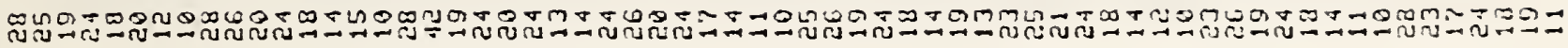

Пே

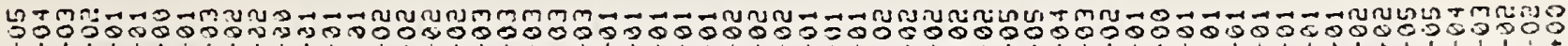

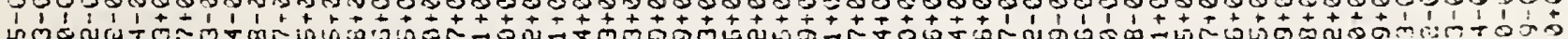

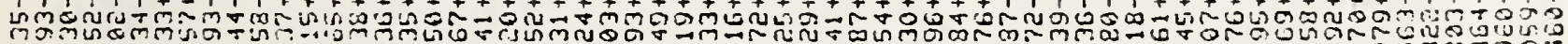

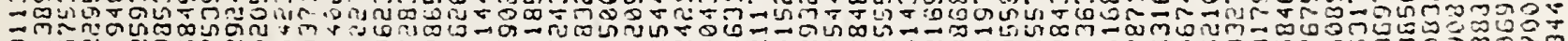
- - -

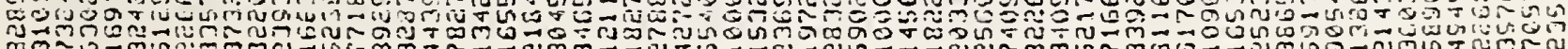

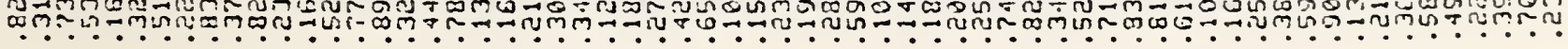

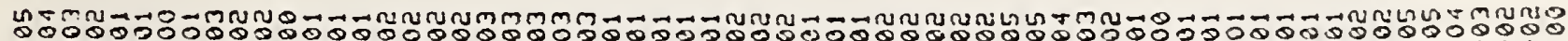

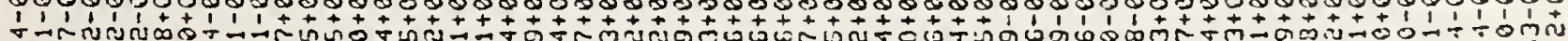
Fี

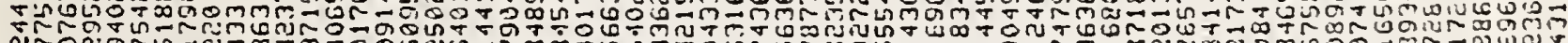

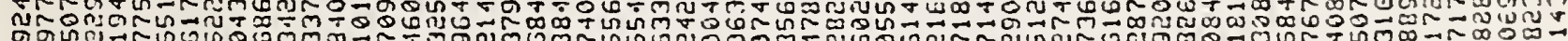

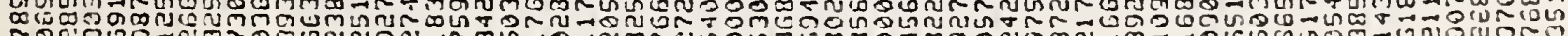
సึ心

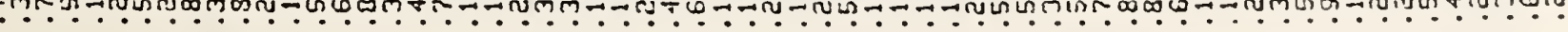


รัญ

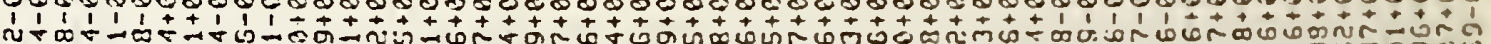

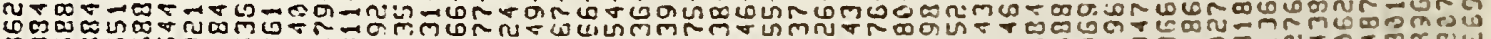
Dond

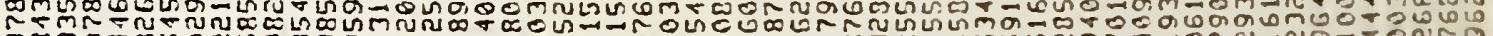

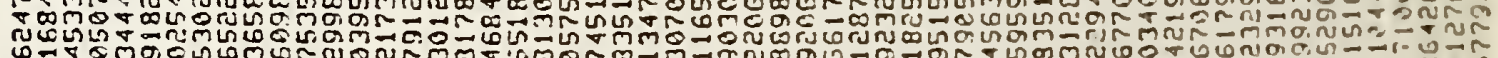

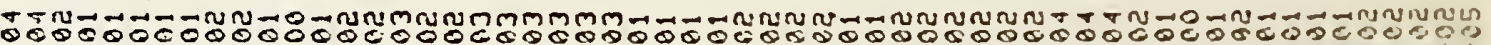

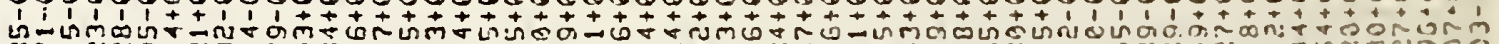
ผ

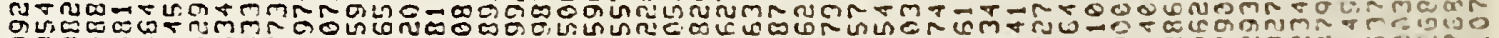
Óm

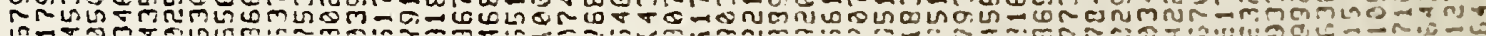

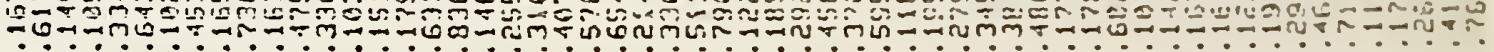

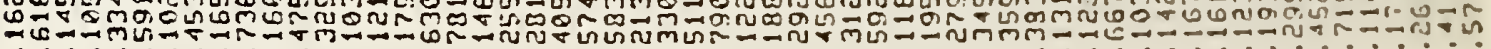

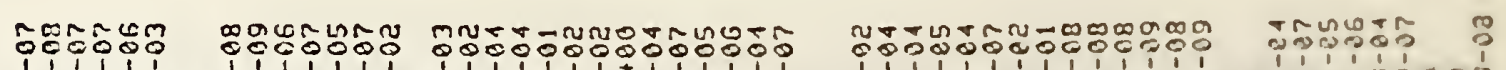

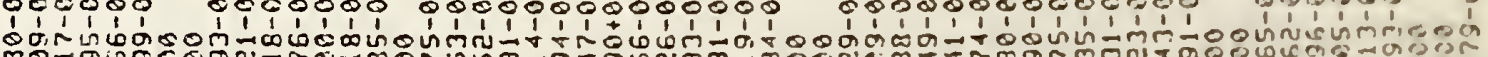

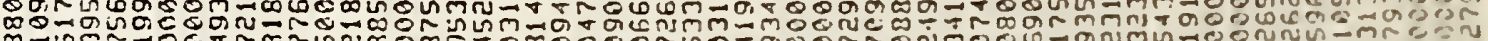

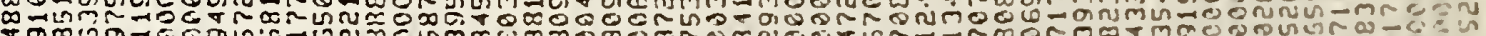

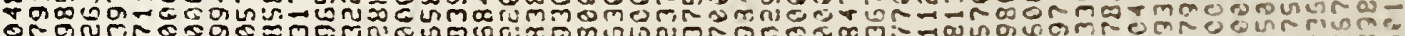

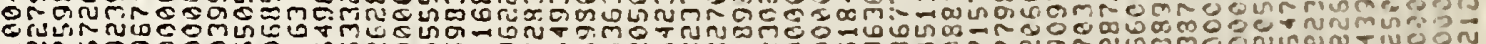

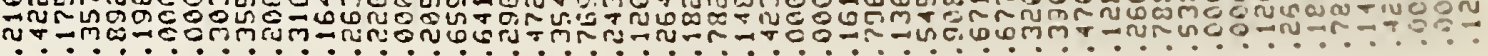




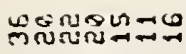

นungentom

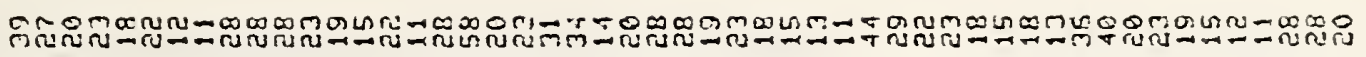

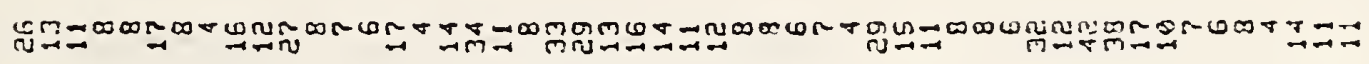

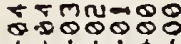

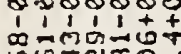

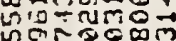

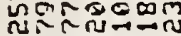
Nowmañ

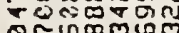

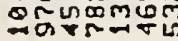

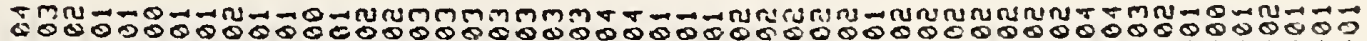

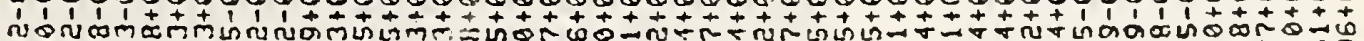
ᄀQ

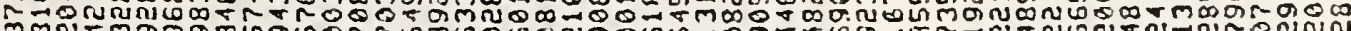
mañogoming mrर mư

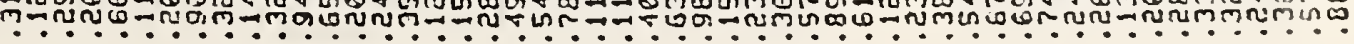

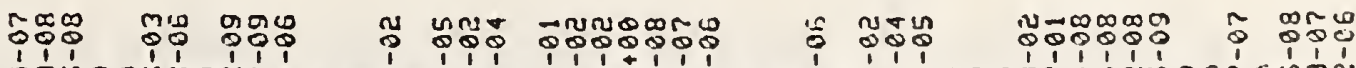
TMno กnnD:N जnष

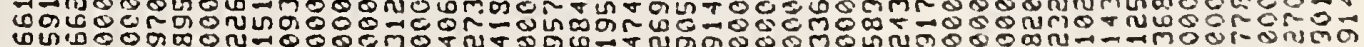
юm: 


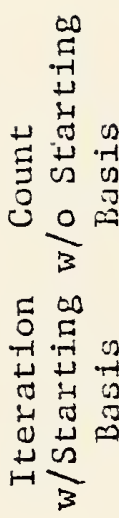
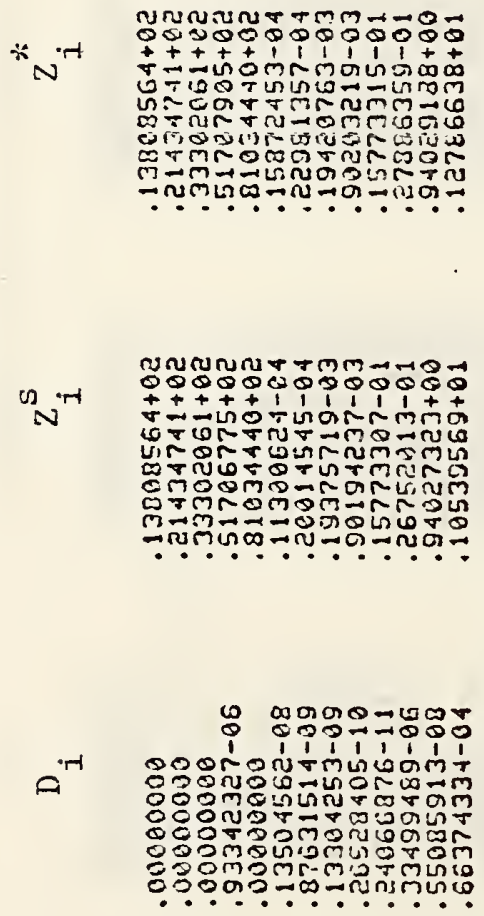

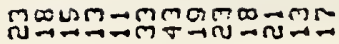

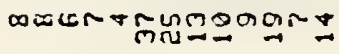

웃ำณ

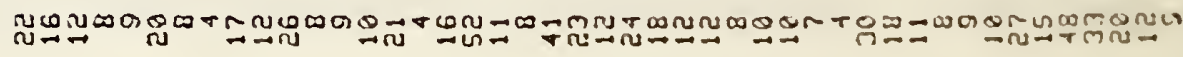
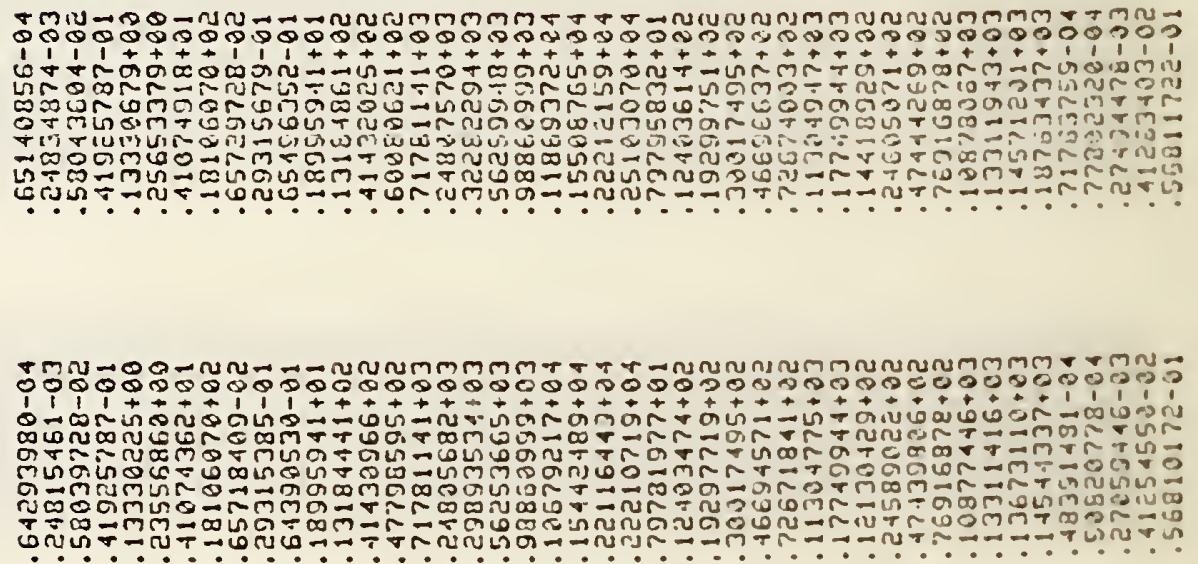

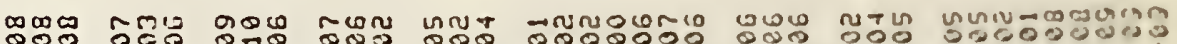

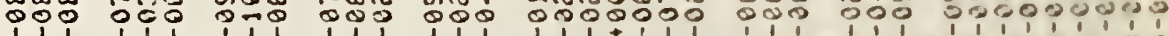

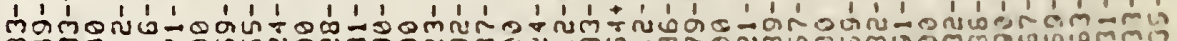

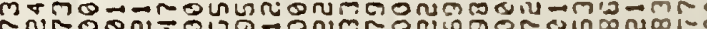
फ

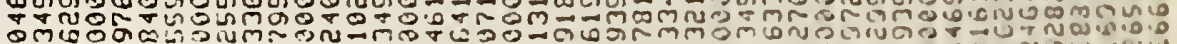
Q

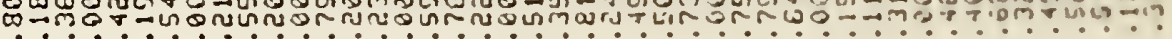




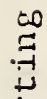

इ

S 0 थ

3

a 00

今

出 Tू-

त क ल

ए

$\stackrel{\infty}{\infty}$

तs 心onching

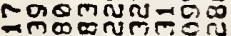

- NRDonot

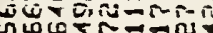

- - -ru-runar $1+-+++4+4$ uññ

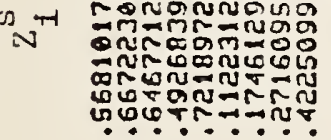

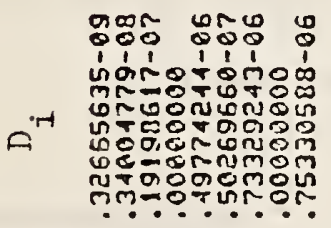


MQS-IILA (REV. Q-78)

U.S. DEPT. OF COMM.

BIBLIOGRAPHIC DATA

SHEET

1. PUBLICATION OR REPORT NO.

NBSIR $86-3389$

Y. Recipient's Accessiont Na.

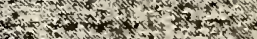

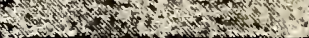

4. TITLE AND SUBTITLE

A Near-optimal Starting Solution for Polynomial Approximation of a Continuous Function in the $L_{1}$ Norm

5. Publication Date

IAY 1986

6. Pertoming Oremization Code

3. Performing Organ. Report No.

7. AUTHOR(S)

Paul D. Domich

9. PERFORMING ORGANIZATION NAME AND ADDRESS

10. Prolect Task/Nork Unit Ma

NATIONAL BUREAU OF STANDARDS

DEPARTMENT OF COMMERCE

11. Contract/Grant No.

WASHINGTON, DC 20234

12. SPONSORING ORGANIZATION NAME AND COMPLETE ADDRESS (Stroot, Clty, state, zIP)

13. Type of Report \& Period Covered

If Sporsoring Agency Code

15. SUPPLEMENTARY NOTES

Document describes a computer program; SF-135, FIPS Software Summary, is attached.

16. ABSTRACT (A 200-word or less fectual summary of most sigrificant information. Il document includee a signilicane bibllography or litorature survey, mention it here.)

This paper presents a method of selecting a near-optimal starting basis for a large class of polynomial approximation problems in the $L_{1}$ norm. While it is possible to prove the optimality of these advanced starting solutions for only a small class of problems, empirical evidence indicates the starting bases are nearly optimal for a much larger class of problems. This paper presents the method used to determine the starting basis and a heuristic justification backed by empirical results supporting its use.

17. KEY WORDS (six to kwolve ontries; alphabetical order; cepitallze only the firet lotter of the liref ker word unlees a proper naele: sopurated by semicolone)

Approximation; Chebyshev polynomials; computational experiment; least absolute deviation; near-optimal starting basis; polynomial approximation

18. AVAILABILITY

E. Unlimited

For Official DIstribution. Do Hot Release to NTIS

Order From Sup. of Doc., U.S. Government Printing Office, Wasinıton, DC 20402, SD Stock No. SNÓO3-003-

X] Order From National Technical Information Service (NTIS), Springfield, VA. 22161

\begin{tabular}{l|c|}
$\begin{array}{l}\text { 19. SECURITY CLASS } \\
\text { (THIS REPORT) }\end{array}$ & $\begin{array}{c}\text { 21. NO. OF } \\
\text { PRINTED PAGES }\end{array}$ \\
$\begin{array}{l}\text { UNCLASSIFIED } \\
\text { (THIS PAGE) } \\
\text { UNECURITY CLASS }\end{array}$ & 27 \\
\hline UNCLASSIFIED & $\$ 9.95$ \\
\hline
\end{tabular}


•

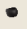


